\title{
Roles and rules of Syngo iFLOW in neuroendovascular procedures
}

\author{
Marco Zenteno ${ }^{1}$, Luis Rafael Moscote-Salazar ${ }^{2}$, Angel Lee ${ }^{3}$ \\ ${ }^{1}$ Professor of Neurological Endovascular Therapy, Departamento de Terapia Endovascular Neurológica, \\ Instituto Nacional de Neurología y Neurocirugía, Universidad Nacional Autónoma de México; \\ StrokeUnit, Hospital Ángeles del Pedregal. \\ ${ }^{2}$ Department of Neurological Endovascular Therapy, Instituto Nacional de Neurología y Neurocirugía; \\ México City, México, mineurocirujano@aol.com \\ ${ }^{3}$ Department of Neurosurgery, Instituto Nacional de Ciencias Médicas y Nutrición Salvador Zubirán; \\ StrokeUnit, Hospital Ángeles del Pedregal, México City, México
}

\begin{abstract}
The authors present 2 patients who underwent neuroendovascular procedures in syngo iFLOW produced that use the dynamic of fluid in several types of intracranial pathologies. As part of a combined CT/angiography suite, iFLOW offered the major advantage of immediate detection or exclusion of intracranial complication without patient transfer. The study of fluid dynamics constitutes a cornerstone for the evaluation of various intracranial vascular pathologies. These applications include the isolation of cerebral aneurysms by embolization and clipping, embolisation of malformations, as well the evaluation of vasooclussive diseases. The emergence of techniques such as syngo iFLOW, which give a comprehensive picture of angiography, constitute a element that can to contribute to the decision of conduct clinics. Siemens has developed a novel based system which is able to reconstruct in achieving angiography techniques colors define intracranial flow characteristics in a single image. With this technique, is possible to obtain a comprehensive picture of cerebral angiography.
\end{abstract}

\section{Technique}

We used a biplane C-arm with flat detector angiography, AXIOM Artis dBA (Siemens, Erlangen, Germany), with a system of dynamic imaging with high resolution flat detector. Spatial and contrast resolution of 14 bits, input plane detector 30 x 40 (48cm diagonal) with a pixel size of $154 \mathrm{um}$, and input level detector of $20 \times 20$ (25 cm diagonal) with a pixel size of $184 \mathrm{um}$ with DSA image acquisition in real time with up to 7.5 imag./s, 10, 30 imag./s in biplane in 1024 x 1024 matrix with a depth of up to 14-bit digitization (7.5 imag./s in matrix of $2,480 \times 1,920)$ rotational angiography DSA Dynavision Dynavision DR and up to 60 imag./s.

The specifications for the processing of the images were: standardized platform with Syngo user interface, simultaneous display of subtraction fluoroscopy/digital subtraction fluoroscopy and original images by Roadmap Plus (digital subtraction fluoroscopy plus); DICOM Print (services printing), DICOM Send /storage commitment (commitment storage/shipping) and DICOM query / Retrieve (search and retrieval) full DICOM functionality in both patient data acquisition and in documentation and archiving, and Dyna CT program (Siemens). 


\section{Case reports}

Case 1

This is a 23 year old male, who presented a seizure, it was performed a brain magnetic resonance, that evidenced a vascular injury in the left parietal region; cerebral angiography was performed, an evidenced a left parietal arteriovenous malformation SM III, see figures 1, 2, 3, 4 . A surgical management was performed. iFLOW was conducted to determine the postoperative absence of injury consistent with postoperative angiography.

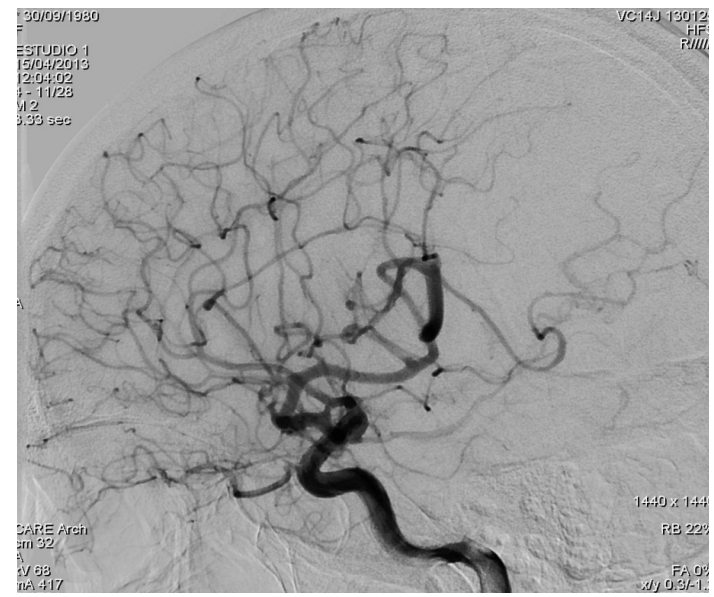

Figure 1

A Lateral cerebral angiogram showing postoperative angiogram showing a complete resection of AVM in parietooccipital lobule

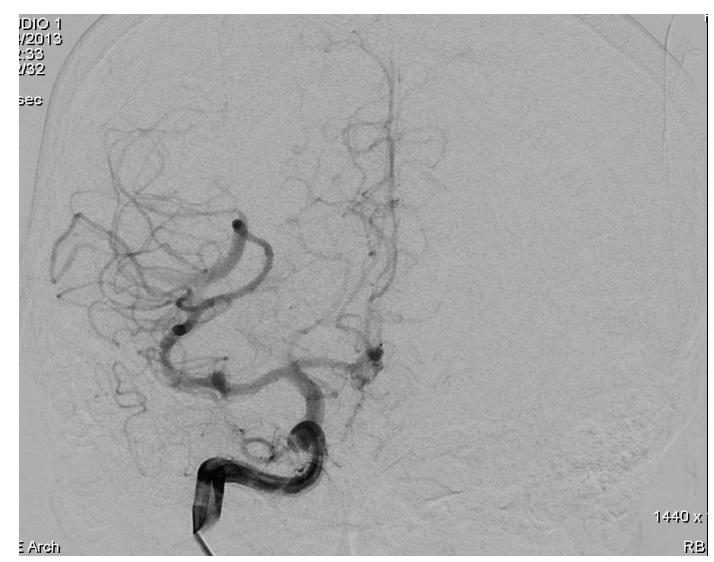

Figure 2

A AP cerebral angiogram showing ausence of AVM

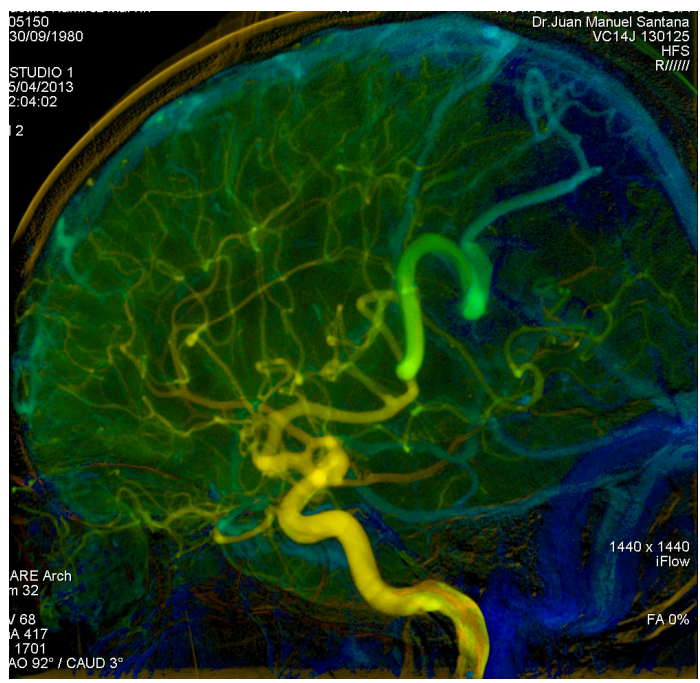

Figure 3

A Lateral Syngo iFlow showing ausence of dynamic of fluid in parietooccipital lobule, confirming the angiographic findings

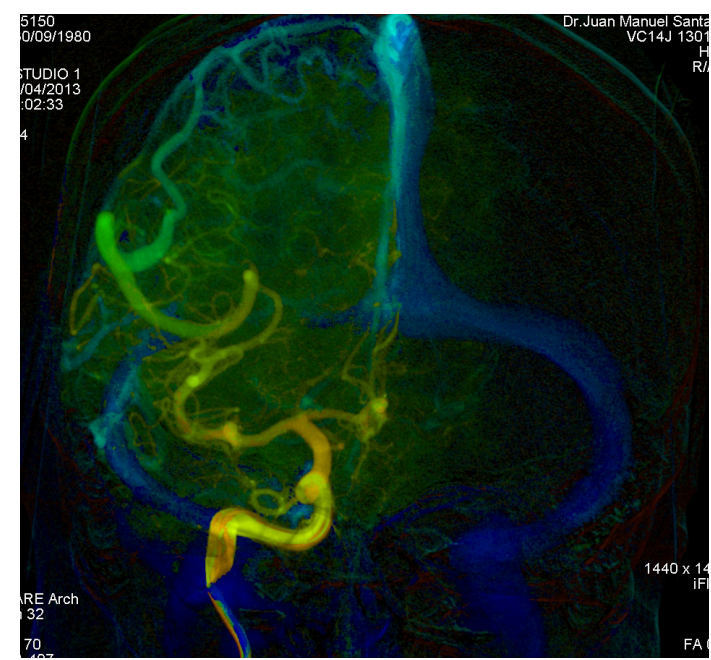

Figure 4

Lateral Syngo iFlow showing ausence of dynamic of fluid in parietooccipital lobule, confirming the angiographic findings

\section{Case 2}

This 35-year old woman, presenting with a grade II Hunt and Hess (H\&H) subarachnoid hemorrhage (SAH), was treated for a rupture of an anterior communicating aneurysm. Cerebral angiography was performed on the 4th day, identifying important postoperative cerebral 
vasospasm, which was handled with chemical thrombolysis, then is performed iFLOW which signals the presence of severe vasospasm. The patient was transferred to the intensive care unit and on awakening from anesthesia, she was found to be neurologically unchanged.

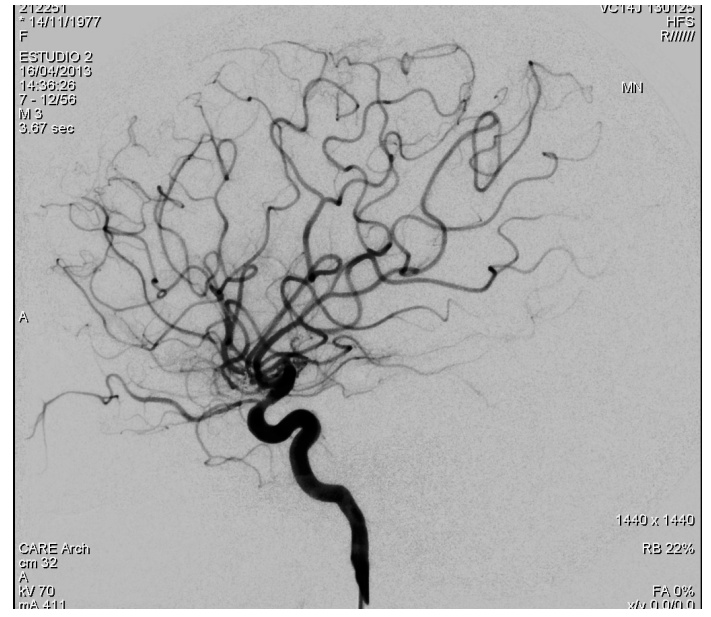

Figure 5

A cerebral angiogram Lateral showing vasoespams in anterior and medial circulation

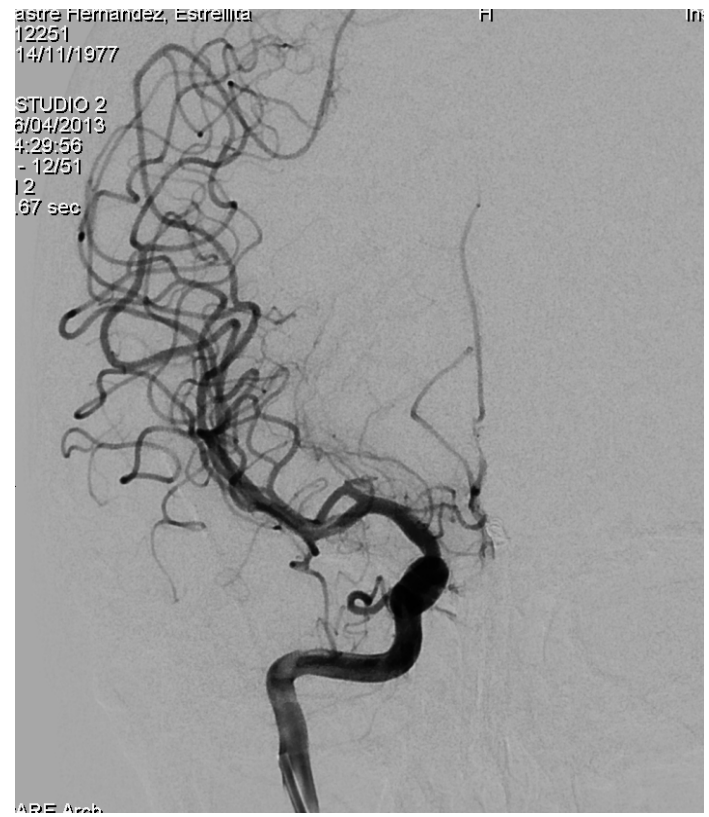

Figure 6

A AP cerebral angiogram Lateral showing vasoespams in anterior and medial circulation

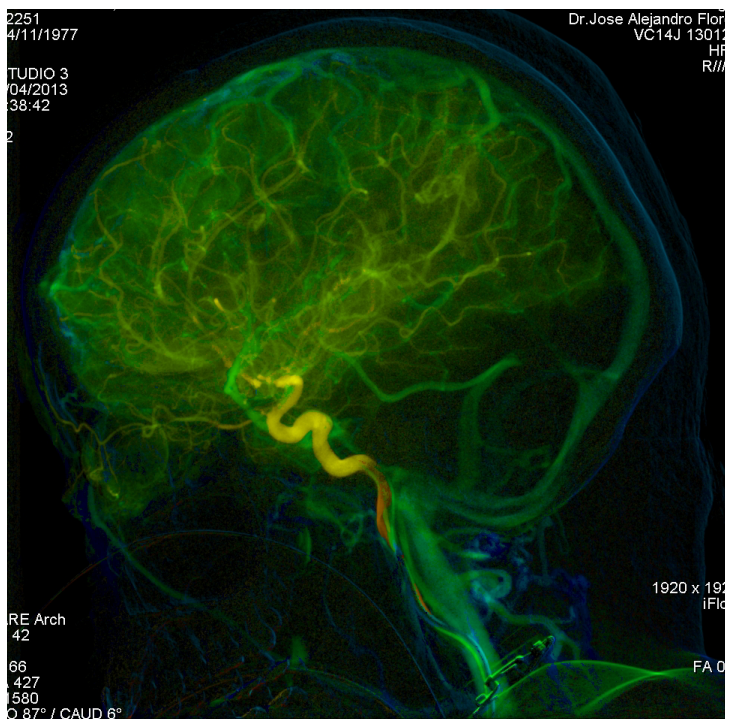

Figure 7

A Lateral Syngo iFLOW showing decrease of dynamic of fluid in the patient

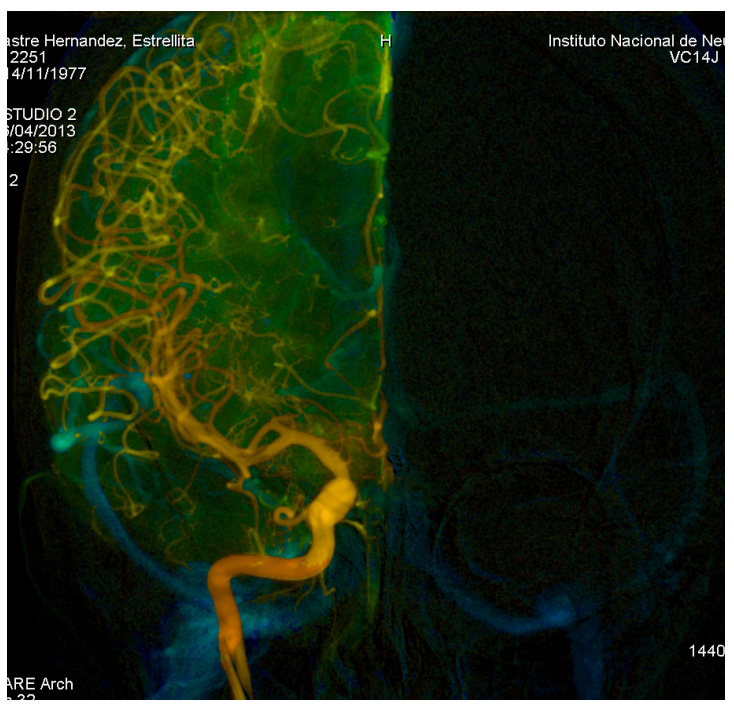

Figure 8

A AP Syngo iFLOW showing decrease of dynamic of fluid in the patient. (Orange)

\section{Discusion}

The assessment of intracranial hemodynamics plays an important role in various pathologies, such as arteriovenous malformations, aneurysms and cerebral malformations. In the case of cerebral aneurysms, various items such as blood 
velocity, the wall shear stress, pressure, particle residence and impact flow are important for growth and rupture of cerebral aneurysms. In the case of conditions such as where there moya moya occlusive stenosis supraclinoid progressive segment of the internal carotid artery flow modification is key to the development of ischemic processes.

The development of imaging technologies has been driven also by the realization of imaging studies with gadolinium and this may be a factor in the development of nephrogenic systemic fibrosis in patients with renal failure,

Nephrogenic Systemic Fibrosis is a rare, first recognized in 1997 and described in the literature since 2000 with about 250 cases reported. His connection as serious adverse event following the administration of contrast agents related to gadolinium in studies of magnetic resonance (MR) performed on patients with advanced stages of kidney failure is of numerous research papers in recent years. (3). Cerebral blood flow can be quantified to allow differentiation characteristics between patients, operators and embolic materials. in the same way provides the ability to quantifythe prognosis of embolization of AVM.

This has generated a growing interest in the realization of angiographic methods using intrinsic methods rather than extrinsic to the image generation, in the same way that the optimization of resources and the creation of cost-effective methods.

\section{Conclusion}

The utilization of Syngo iFLOW allows the determination of the flow dynamics in intracerebral vascularized lesions, its usefulness lies in its ability to determine the presence or absence of blood flow, a key for the management of vascular lesions amenable to surgical handling and mainly neuroendovascular.

\section{Acknowledgments}

The authors thank our outstanding radiology technologists who have worked tirelessly to improve iFLOW images images: Susana Vega Montesinos.

\section{Correspondence}

Dr. Luis Rafael Moscote, Universidad de Cartagena, Cartagena de Indias, e-mail: mineurocirujano@aol.com

\section{References}

1. Chatziprodromou I, Tricoli A, Poulikakos D, Ventikos Y. Haemodynamics and wall remodelling of a growing cerebral aneurysm: a computational model. J Biomech. 2007; 40(2):412-26.

2. Watton PN, Raberger NB, Holzapfel GA, Ventikos Y. Coupling the hemodynamic environment to the evolution of cerebral aneurysms: computational framework and numerical examples. J Biomech Eng. 2009 Oct;131(10):101003

3.Issa N, Poggio ED, Fatica RA, Patel R, et al. Nephrogenic systemic fibrosis and its association with gadolinium exposure during MRI. Cleveland Clinic Journal of Medicine 2008;75(2):95-111. 